\title{
Proteome of breast cancer derived microvesicles
}

\author{
Y. Risha ${ }^{1}$, V. Susevski ${ }^{1}$, N. Hüttmann ${ }^{1}$, S. Poolsup ${ }^{1}$, Z. Minic ${ }^{2}$, M. V. Berezovski ${ }^{1,2}$ \\ ${ }^{1}$ Department of Chemistry and Biomolecular Sciences, University of Ottawa, Ottawa ON K1N 6N5, Canada \\ ${ }^{2}$ John L. Holmes Mass Spectrometry Facility, Faculty of Science, University of Ottawa, Ottawa ON K1N 6N5, Canada
}

The aim of the research. To examine the proteomic profile of breast cancer exosomes.

Material and methods. Cell lines used for this study were MDA-MB-231 female epithelial breast cancer cells (ATCC HTB-26) and MCF10A non-tumorigenic epithelial breast tissue cells. MVs were isolated using differential ultracentrifugation. Samples were lysed, reduced, alkylated, digested, and analyzed by an Orbitrap Fusion mass spectrometer. MS raw files were analyzed using MaxQuant version 1.6.12.0. Peptides were searched against the human UniProt FASTA database using the Andromeda search engine, integrated into MaxQuant.

Results. MVs derived from MCF10A and MDA-MB-231 cell lines were analyzed, and 1427 and 547 proteins were identified in the MDA-MB-231 and MCF10A-derived MVs, respectively. In total, 455 proteins were common to both MDA-MB-231 and MCF10A MVs. MVs derived from MCF10A and MDAMB-231 cell lines were analyzed, and 1427 and 547 proteins were identified in the MDA-MB-231 and MCF10A-derived MVs, respectively. In total, 455 proteins were common to both MDA-MB-231 and MCF10A MVs. The unique MDA-MB-231 MV proteins were searched against the DisGeNET human diseases database. Out of 972 MDA-MB-231 MV proteins, 112 were cancer-related while 32 were specifically associated with BC. In the MDA-MB-231 MV proteome, 23 Wnt signaling pathway proteins were identified based on their GO biological process. Proteomic analysis identified enzymes OAT, TALDO1, and BLMH were only in MVs from metastatic MDA-MB-231 cell line. The specific activity of OAT and TALDO1 was higher in MV fractions of MDA-MB-231 in comparison to the non-cancerous MCF10A cell line-derived MVs. These findings might suggest that these enzymes might play a role in BC. In our present study, we found that some enzymes identified from MV fractions were already proposed to play a role in cancer therapy as therapeutic targets (OAT, TALDO1) and resistance against chemotherapy agents (BLMH).

Key words: proteomic analysis, breast cancer, ATCC HTB-26.

Conclusion. We found that aminotransferase enzyme, transaldolase, and bleomycin hydrolase are specific for MDA-MB-231 MVs.

Conflict of interest. The authors declare the absence of obvious and potential conflicts of interest associated with the publication of this article.

Citation: Risha Y, Susevski V, Hüttmann N, Poolsup S, Minic Z, Berezovski MV. Proteome of breast cancer derived microvesicles. Siberian Medical Review. 2021; (2):68-71. DOI: 10.20333/2500136-2021-2-68-71

\section{Introduction}

The development of metastatic BC is characterized by certain hallmarks modulation of microenvironment, angiogenesis, sustained growth and tissue invasion that rely on cell-cell signaling and communication [1,2]. Extracellular vesicles (EVs) have emerged as a novel mechanism of intercellular communication that does not require direct cellular contact [3]. EVs may be classified based on their morphology, cellular origin, or biogenesis pathways. Small EVs (sEVs), including exosomes (Exo), range in size from 30 to $150 \mathrm{~nm}$ $[4,5]$. On the other hand, microvesicles (MVs) are formed by direct outward budding of the cell membrane and are $50 \mathrm{~nm}$ to $>1000 \mathrm{~nm}$ in diameter [6]. In this study, vesicles of a size between 100 and $500 \mathrm{~nm}$ are referred to as MVs.

Small EVs play an important role in cancer progression by supporting tumor metastasis, drug resistance, and immune system evasion [7]. Similarly, MVs contribute to cancer progression; however, their precise role is less understood [8]. MVs also play important roles in BC through the transfer of oncogenic molecules. MVs secreted from multi-drug resistant $\mathrm{BC}$ cells can aid in immune evasion by transforming macrophages to an incapacitated state [9]. The global study of proteins using modern proteomic methods has provided a deeper understanding of many diseases [10]. EV proteomics can elucidate the biological role of EVs in BC progression and therapy.
MV proteomic profiling elucidated possible drug resistance mechanisms in BC through the transfer and stabilization of P-glycoprotein [11]. In this study, the proteomes of the metastatic BC cell line MDA-MB-231-derived MVs were compared to the non-cancerous cell line MCF10A-derived MVs. Differences were identified in the MV proteins when compared to the previously profiled sEV proteome derived from the same metastatic BC cell line MDA-MB-231 [12]. There are significant differences in the proteomes of BC-derived exosomes and MVs. Three enzymes unique to MDA-MB-231 were validated in cells, sEVs, and MVs.

\section{Material and methods}

Cell lines used for this study were MDA-MB-231 female epithelial breast cancer cells (ATCC HTB-26) and MCF10A non-tumorigenic epithelial breast tissue cells (ATCC CRL10317). Cells were grown in complete media. When cells reached 40-50 \% confluency, they were rinsed with Dulbecco modified phosphate buffered saline (PBS) and incubated with vesicle depleted media for $48 \mathrm{~h}$. Vesicle depleted media was prepared by ultracentrifugation of FBS or HS at $100,000 \times \mathrm{g}$ for $20 \mathrm{~h}$. The vesicle-enriched media was then collected and processed for the isolation of MVs.

MVs were isolated using differential ultracentrifugation. Then, $100 \mathrm{~mL}$ of cell culture supernatant were collected and immediately centrifuged at $300 \times \mathrm{g}$ for $10 \mathrm{~min}$. 
This was followed by a $3000 \times \mathrm{g}$ spin on a Sigma13190 rotor (MBI) for $10 \mathrm{~min}$, then a $15,000 \times \mathrm{g}$ centrifugation for 35 min using an SW28 swinging bucket rotor (Beckman Coulter). The MV-containing pellet was collected after the $16,000 \times \mathrm{g}$ spin in PBS. The collected MVs were washed by centrifuging at $15,000 \times \mathrm{g}$ for $1 \mathrm{~h}$ using an SW55Ti swinging bucket rotor (Beckman Coulter). The sedimented MVs were finally resuspended in PBS.

Samples were lysed, reduced, alkylated, digested, and analyzed by an Orbitrap Fusion mass spectrometer (Thermo Fisher Scientific, Mississauga, ON, Canada) coupled to an UltiMate 3000 nanoRSLC (Thermo Fisher Scientific, Mississauga, ON, Canada) as described previously [11]. MS raw files were analyzed using MaxQuant version 1.6.12.0 [13]. Peptides were searched against the human UniProt FASTA database using the Andromeda search engine [14], integrated into MaxQuant.

\section{Results and discussion}

MVs derived from MCF10A and MDA-MB-231 cell lines were analyzed, and 1427 and 547 proteins were identified in the MDA-MB-231 and MCF10A-derived MVs, respectively. In total, 455 proteins were common to both MDA-MB-231 and MCF10A MVs. Common proteins were excluded from the GO annotations (biological process and cellular localization) enrichment analysis. The "extracellular exosome" cellular localization label was highly enriched in both proteomes. Enrichment of mitochondrial proteins was found in the MCF10A MV proteome. This was evident by cellular localization terms such as "mitochondrion", "mitochondrial inner membrane" and "mitochondrial respiratory chain complex III." Biological process terms identified from the MCF10A MV proteome supported the enrichment of mitochondrial proteins with terms that included "mitochondrial electron transport, ubiquitin to cytochrome c", "aerobic respiration" and "hydrogen ion transport". The MDA-MB-231 MV proteins mainly originated from the cytosol, membrane, and ribosome according to their cellular localization terms. The GO cellular localization term "mitochondrion" was also identified with a $\mathrm{p}$-value of $1.3 \times 10-2$. Biological process terms related to the MDA-MB-231 MV proteome included terms related to translation: "translation initiation" and "SRP-dependent co-translational protein targeting to membrane". Energy-related biological process terms. Nanoparticle tracking analysis (NTA) characterization showing size distributions of isolated microvesicles (MVs) from (A) MCF10A and (B) MDA-MB-231 cell lines.

MVs derived from MCF10A and MDA-MB-231 cell lines were analyzed, and 1427 and 547 proteins were identified in the MDA-MB-231 and MCF10A-derived MVs, respectively. In total, 455 proteins were common to both MDA-MB-231 and MCF10A MVs. Common proteins were excluded from the GO annotations (biological process and cellular localization) enrichment analysis. The "extracellular exosome" cellular localization label was highly enriched in both proteomes. Enrichment of mitochondrial proteins was found in the MCF10A MV proteome. This was evident by cellular localization terms such as "mitochondrion", "mitochondrial inner membrane" and "mitochondrial respiratory chain complex III." Biological process terms identified from the MCF10A MV proteome supported the enrichment of mitochondrial proteins with terms that included "mitochondrial electron transport, ubiquitin to cytochrome c", "aerobic respiration" and "hydrogen ion transport". The MDA-MB-231 MV proteins mainly originated from the cytosol, membrane, and ribosome according to their cellular localization terms. The GO cellular localization term "mitochondrion" was also identified with a $\mathrm{p}$-value of $1.3 \times 10-2$. Biological process terms related to the MDA-MB-231 MV proteome included terms related to translation: "translation initiation" and "SRP-dependent co-translational protein targeting to membrane". Energy-related biological process terms included "ATP hydrolysis coupled proton transport" with a p-value of $7.8 \times 10-3$ and "positive regulation of ATPase activity" with a p-value of $2.7 \times 10-2$. The Wnt and NF-kappaB pathways were also represented with terms such as "Wnt signaling pathway, planar cell polarity pathway" and "NIK/ NF-kappaB signaling".

The unique MDA-MB-231 MV proteins were searched against the DisGeNET human diseases database. Out of 972 MDA-MB-231 MV proteins, 112 were cancer-related while 32 were specifically associated with $\mathrm{BC}$. In the MDAMB-231 MV proteome, 23 Wnt signaling pathway proteins were identified based on their GO biological process, 6 of 15 five of which-Rho associated coiled-coil containing protein kinase 2 (ROCK2), casein kinase 2 alpha 1 (CSNK2A1), casein, kinase 2 alpha 2 (CSNK2A2), catenin beta 1 (CTNNB1), and glycogen synthase kinase 3 beta (GSK3B)-have a known cancer association. The DisGeNET identified cancer proteins also included five eukaryotic translation initiation factors (EIFs), three of which were BC associated (Eukaryotic translation initiation factor 1A, X-chromosomal (EIF1AX), Eukaryotic initiation factor 4A-II (EIF4A2), and Eukaryotic translation initiation factor 5A-1 (EIF5A)). Moreover, eleven kinases were cancer-associated, some of which include FYN proto-oncogene tyrosine kinase (FYN), aurora kinase $B$ (AURKB), and integrin-linked kinase (ILK).

In this study, sEV and MV proteins from MDA-MB-231 and MCF10A cell lines were compared using data from a previous study [12]. In total, 372 proteins were common to all EV fractions. There is also a notable overlap of 1272 proteins between MV and sEVs derived from the MDAMB-231 cell line. Finally, 89 proteins were unique to MDAMB-231-derived MVs.

In total, 603 proteins were only identified in sEVs and MVs derived from the MDAMB-231 cell line. To shed light on the quantitative difference of these proteins, fold change and p-value were calculated for 420 proteins with complete observations. In total, 50 and 59 proteins were significantly more abundant $(\mathrm{p}<0.05)$ in sEVs and MVs, respectively. Copine-8 (CPNE8) and calreticulin (CALR) were selected based on differential expression between MDA-MB-231 MVs and sEVs for flow cytometry (FC) analysis. The Venn diagram shows the number of overlapping proteins 
between the different extracellular vesicle (EV) fractions. In total, 603 proteins were only identified in sEVs and MVs derived from the MDA-MB231 cell line. To shed light on the quantitative difference of these proteins, fold change and $\mathrm{p}$-value were calculated for 420 proteins with complete observations. In total, 50 and 59 proteins were significantly more abundant $(\mathrm{p}<0.05)$ in sEVs and MVs, respectively. Copine-8 (CPNE8) and calreticulin (CALR) were selected based on differential expression between MDA-MB-231 MVs and sEVs for flow cytometry (FC) analysis. Vesicle-containing cell supernatant was depleted of cells, debris, and larger vesicles before staining with antibodies. Two populations with relative scattering differences confirmed the presence of CPNE8 and CALR on MDA-MB-231-derived EVs. The positive populations are no longer present after treatment of the supernatant with Triton X-100, hence confirming the vesicle nature of identified populations.

Proteomic analysis identified enzymes OAT, TALDO1, and BLMH were only in MVs from metastatic MDAMB-231 cell line. The specific activity of OAT and TALDO1 was higher in MV fractions of MDA-MB-231 in comparison to the non-cancerous MCF10A cell line-derived MVs. These findings might suggest that these enzymes might play a role in $\mathrm{BC}$.

The OAT enzyme is a mitochondrial protein found in almost all eukaryotic cells. This enzyme converts amino acid L-ornithine and $\alpha$-ketoglutarate to L-glutamate-5-semialdehyde and L-glutamate. The substrate of OAT, ornithine, is a key substrate for the synthesis of proline, polyamines, and citrulline. Ornithine also plays an important role in the regulation of several metabolic processes leading to diseases like hyperornithinemia, hyperammonemia, gyrate atrophy, and cancer in humans [15]. High levels of ornithine have been reported to be a potential protective factor for $\mathrm{BC}$ [16]. Similarly, as we demonstrated for metastatic MDAMB-231 cell line, OAT is overexpressed in hepatocellular carcinoma, and the inhibition of this enzyme has been suggested to be an effective therapy in mice $[17,18]$. Moreover, proteomics analysis using two-dimensional differential gel electrophoresis (2D-DIGE) and matrix-assisted laser desorption/ionization-time-of-flight-mass spectrometry (MALDITOF-MS) of canine mammary tumors, proposed as a model for human breast cancer, identified OAT as one of several upregulated proteins in metastatic carcinomas [19]. In addition, OAT was found upregulated, 1.3-fold, in small cell lung cancer cell line (NCIH446) compared to the non-cancerous human bronchial epithelial cell line (16HBE) [20]. This enzyme's expression has been correlated to the pathological grade and clinical tumor metastasis stage in lung cancer patients $[20,21]$. Furthermore, OAT knockout nude mice exhibited significantly suppressed growth and metastasis of lung cancer xenografts [21]. Metastatic MDA-MB-231 cell line-derived MVs exhibiting higher enzymatic activity of OAT than MVs of the non-cancerous MCF10A cell line suggest that cancer-derived MVs may contribute to the overall metabolic status and consequently increase vulnerability in BC. TALDO1 is an enzyme of the pentose phosphate pathway (PPP) and has been linked to oxidative stress, inflammation, and carcinogenesis [22]. This enzyme transfers threecarbon groups from sedoheptulose-7-phosphate (S7P) to glyceraldehyde-3-phosphate (G3P) to generate erythrose-4-phosphate (E4P) and fructose-6-phosphate (F6P). In fastdividing cancer cells, this enzyme mediates the PPP that plays a pivotal role in helping glycolytic cancer cells meet their anabolic demands and combat oxidative stress [23]. The differential proteomics of urinary EV proteins showed TALDO1 to be associated with bladder cancer [24]. In hepatomas, TALDO1 activity was increased by 1.5-3.4 times compared to normal liver tissue regardless of tumor stage [25]. TALDO1 is indicative of hepatocellular carcinoma metastasis. Expression levels of TALDO1 were higher in the serum of metastatic hepatocellular carcinoma patients compared to controls [26]. Certain genetic polymorphisms in TALDO1 are associated with squamous cell carcinoma of the head and neck [27]. TALDO1 may also play a role in cancer drug resistance; higher levels of TALDO1 expression were associated with poor response to BC HER2 inhibitors in BC patients. The suppression of TALDO1 increased susceptibility to HER2 inhibition cell Biomedicines 2021, 9, 10711 of 15 lines with HER2 amplification [28]. It has been proposed that TALDO1 can potentially be exploited as a biomarker or target for combination therapy in BC. We demonstrated that the specific activity of TALDO1 in cellular protein extracts and MV fractions of MDA-MB-231 was higher than its activity in MV fractions of non-cancerous MCF10A cell line. Our results support reported findings, and that TALDO1 might be a potential therapeutic target in BC. BLMH is a cysteine aminopeptidase that was discovered for its ability to inactivate bleomycin [29]. Bleomycin is a small glycopeptide antibiotic used in combination therapy for the treatment of Hodgkin's lymphoma, non-Hodgkin's lymphoma, testicular cancer, ovarian cancer, and cervical cancer [30]. The antitumor effect of bleomycin is most likely caused by its ability to bind to DNA and induce the formation of toxic DNA lesions via a free radical [31]. Bleomycin hydrolase and poly (ADP-ribose) polymerase-1 are reported to participate in the Ubc9-mediated resistance against chemotherapy agents in human breast carcinoma MCF-7 cells [32]. Our results demonstrate a higher expression of BLMH in cancerous MDA-MB-231 MVs compared to MCF10A MVs. These results indicate that MVs may play a role in bleomycin resistance in $\mathrm{BC}$.

This study and previously published work [12,33] identified the differences in the proteome between cancerous and non-cancerous breast cell line-derived EVs. We identified 87 and 112 proteins from sEVs [12] and MVs, respectively, that are related to cancer. This discovery presents a framework for future in-depth studies of each of these proteins from cancer-derived EVs to investigate whether they may promote cancer progression, invasion, and metastasis, remodeling of the tumor microenvironment, or angiogenesis. Such research can lead to a better understanding of the role of EVs in cancer biology. Moreover, some of these candidate proteins can be later explored for their efficacy 
in diagnostic or therapeutic applications for BC. The big challenge is to implement the identified candidate proteins in clinical samples by further validation and verification in large prospective studies [34]. In our present study, we found that some enzymes identified from MV fractions were already proposed to play a role in cancer therapy as therapeutic targets (OAT, TALDO1) and resistance against chemotherapy agents (BLMH). Future goals are to extensively examine and validate whether the MV enzymes could be transferred while maintaining biological activity and to investigate their biological roles in recipient cells by eventually causing physiological changes. Following this approach, EVs may serve as a source of bioactive proteins that can be used in cancer therapy, as already proposed for the treatment of several diseases [35].

\section{Conclusion}

We found that aminotransferase enzyme (OAT), transaldolase (TALDO1), and bleomycin hydrolase (BLMH) are specific for MDA-MB-231 MVs.

\section{References}

1. The Global Cancer Observatory (GCO), Cancer Today. IARC, Lyon. 2018. Accessed October 11, 2019. https://gco.iarc.fr.

2. Hendrick RE, Baker JA, Helvie MA. Breast Cancer Deaths Averted over 3 Decades. Cancer. 2019;(125): 1482-1488.

3. Carioli G, Malvezzi M, Rodriguez T, Bertuccio P, Negri E, Vecchia CL. Trends and Predictions to 2020 in Breast Cancer Mortality in Europe. Breast. 2017; (36):89-95.

4. Mariotto AB, Etzioni R, Hurlbert M, Penberthy L; Mayer M. Estimation of the Number of Women Living with Metastatic Breast Cancer in the United States. Cancer Epidemiology Biomarkers \& Prevention. 2017;(26):809-815.

5. Calvo F, Sahai E. Cell Communication Networks in Cancer Invasion. Current Opinion in Cell Biology. 2011; (23):621-629.

6. Kapoor P, Saunders MM, Li Z, Zhou Z, Sheaffer N, Kunze EL, Samant RS, Welch DR, Donahue HJ. Breast Cancer Metastatic Potential: Correlation with Increased Heterotypic Gap Junctional Intercellular Communication between Breast Cancer Cells and Osteoblastic Cells. International Journal of Cancer. 2004;(111):693-697.

7. Shah R, Patel T, Freedman JE. Circulating Extracellular Vesicles in Human Disease. The New England Journal of Medicine. 2018;(379):958-966.

8. Huang $X$, Yuan T, Tschannen M, Sun Z, Jacob H, Du M, Liang M, Dittmar RL, Liu Y, Liang M. Characterization of Human Plasma-Derived Exosomal RNAs by Deep Sequencing. BMC Genomics. 2013;(14):319.

9. Carvalho AS, Henrique Baeta H, Moraes MCS, Beck HC, Rodriguez MS, Saraswat M, Pandey A, Matthiesen R. Extra-Cellular Vesicles Carry Proteome of Cancer Hallmarks. Frontiers in Bioscience. 2020;(25):398-436.

10. Brzozowski JS, Jankowski H, Bond DR, Mccague SB, Munro BR, Predebon MJ, Scarlett CJ, Skelding KA,
Weidenhofer J. Lipidomic Profiling of Extracellular Vesicles Derived from Prostate and Prostate Cancer Cell Lines. Lipids in Health and Disease. 2018;(17):211.

11. Xu R, Greening DW, Zhu H-J, Takahashi N, Simpson RJ. Extracellular Vesicle Isolation and Characterization: Toward Clinical Application. Journal of Clinical Investigation. 2016;(126):1152-1162.

12. Minciacchi VR, Freeman MR, Vizio DD. Extracellular Vesicles in Cancer: Exosomes, Microvesicles and the Emerging Role of Large Oncosomes. Seminars in Cell and Developmental Biology. 2015;(40):41-51.

13. Vizio DD, Morello M, Dudley AC, Schow PW, Adam RM, Morley S, Mulholland D, Rotinen M, Hager MH, Insabato L. Large Oncosomes in Human Prostate Cancer Tissues and in the Circulation of Mice with Metastatic Disease. The American Journal of Pathology. 2012;(181):1573-1584.

14. Li I, Nabet BY. Exosomes in the Tumor Microenvironment as Mediators of Cancer Therapy Resistance. Molecular Cancer. 2019;(18):1-10.

15. Jayaseelan VP. Emerging Role of Exosomes as Promising Diagnostic Tool for Cancer. Cancer Gene Therapy. 2019;(27):395-398.

16. Wu C-Y, Du S-L, Zhang J, Liang A-L, Liu Y-J. Exosomes and Breast Cancer: A Comprehensive Review of Novel Therapeutic Strategies from Diagnosis to Treatment. Cancer Gene Therapy. 2016;(24):6-12

17. Li X, Wang Y, Wang Q, Liu Y, Bao W, Wu S. Exosomes in Cancer: Small Transporters with Big Functions. Cancer Letters. 2018;(435):55-65.

18. An M, Zhu J, Wu J, Cuneo KC, Lubman DM. Circulating Microvesicles from Pancreatic Cancer Accelerate the Migration and Proliferation of PANC-1 Cells. Journal of Proteome Research. 2018;(17):1690-1699.

19. Huaitong X, Yuanyong F, Yueqin T, Peng Z, Wei S, Kai S. Microvesicles Releasing by Oral Cancer Cells Enhance Endothelial Cell Angiogenesis via Shh/RhoA Signaling Pathway. Cancer Biology \& Therapy. 2017;(18):783-791.

20. Menck K, Bleckmann A, Wachter A, Hennies B, Ries L, Schulz M, Balkenhol M, Pukrop T, Schatlo B, Rost U. Characterisation of Tumour-Derived Microvesicles in Cancer Patients' Blood and Correlation with Clinical Outcome. Journal of Extracellular Vesicles. 2017;(6):1340745.

\section{Author information}

Yosef Risha, Researcher, PhD, Department of Chemistry and Biomolecular Sciences, University of Ottawa, Ottawa, ON K1N 6N5, Canada; e-mail: yrish083@uottawa.ca

Vanessa Susevski, Researcher, PhD, Department of Chemistry and Biomolecular Sciences, University of Ottawa, Ottawa, ON K1N 6N5, Canada; e-mail: vsuse027@uottawa.ca

Nico Hüttmann, Researcher, PhD, Department of Chemistry and Biomolecular Sciences, University of Ottawa, Ottawa, ON K1N 6N5, Canada; e-mail:nhutt069@uottawa.ca

Suttinee Poolsup, Researcher, PhD, Department of Chemistry and Biomolecular Sciences, University of Ottawa, Ottawa, ON K1N6N5, Canada; e-mail: spool093@uottawa.ca

Zoran Minic, Researcher, PhD, John L. Holmes Mass Spectrometry Facility, Faculty of Science, University of Ottawa, Ottawa, ON K1N 6N5,Canada; E-mail: zminic@uottawa.ca

Maxim V Berezovski, Professor, PhD, Department of Chemistry and Biomolecular Sciences, University of Ottawa, Ottawa, ON K1N 6N5, Canada; 2John L. Holmes Mass Spectrometry Facility, Faculty of Science, University of Ottawa, Ottawa, ON K1N 6N5, Canada; e-mail:Maxim.Berezovski@uottawa.ca 